\title{
The association between salt intake and obesity in UK adults aged 19-64 years
}

\author{
R. Lydon ${ }^{1}$, L. McAnena ${ }^{2}$, M.B.E Livingstone ${ }^{2}$ and M.A. Kerr ${ }^{2}$ \\ ${ }^{1}$ Home Economics Department, St. Angela's College, Lough Gill, Sligo, Republic of Ireland and ${ }^{2}$ Northern Ireland \\ Centre for Food and Health (NICHE), Ulster University, Coleraine
}

High salt intake (SI) is an established risk factor for several chronic diseases including hypertension, stroke and cardiovascular disease. Less well documented is evidence reporting salt as a potential risk factor for the development of obesity ${ }^{1-3}$. Whether or not the relationship between salt and obesity is dependent or independent of energy intake (EI) remains unconfirmed. Consequently, this study aimed to investigate the association between SI and adiposity measures amongst a representative sample of UK adults aged 19-64 years using National Dietary and Nutritional Survey data (NDNS, 2014). Dietary intake from 4-day estimated food diaries and anthropometric measurements (body mass index (BMI), waist circumference (WC), waist-hip ratio (WHR) and \% body fat) were examined in relation to measured SI's from 24-hour urinary sodium excretion (NaEx) (n 605; 279 males; 326 females).

A significant positive correlation was found between SI and all measures of body composition available, with the strongest correlation for BMI after adjusting for age, gender, smoking, alcohol, misreporting, EI, ethnic group and socioeconomic status $(P<0 \cdot 001, r$ $=.201)$. BMI and WC increased significantly across quartiles of sodium density (salt intake/kJ) for both males and females after controlling for misreporting of EI and age (Figure 1).
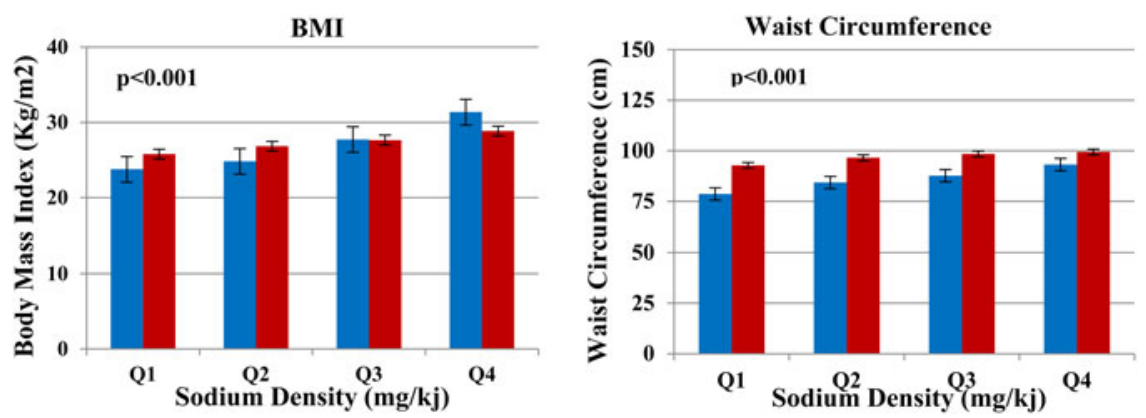

Fig. 1. Median BMI and WC according to sodium density (SD) ${ }^{a}$ (derived from NaEx values; mg/kj) in males and females.* Males Females * Multivariable linear regression analyses adjusted for age and misreporting ${ }^{\mathrm{a}}$ Cut off values for quartiles of SD (mg/kj): Q1, .02-.26; Q2, .26-.37; Q3, $.37-.50 ; \mathrm{Q} 4, .50-1.66)$ Bars represent median values $( \pm \mathrm{SE})$.

The current findings suggest that high SIs are associated with an increased risk of obesity, irrespective of EI. The relationship between adiposity and SI may only be possible to address via randomised controlled trials comparing response to varying SIs in lean versus obese participants. Meanwhile, the current findings may provide scope for public health interventions to focus particularly on reducing SI in overweight/obese individuals.

1. Grimes CA, Bolhuis DP, Feng HJ et al. (2016) Br J Nutr 5:7.

2. Ma Y, He FJ \& MacGregor GA (2015) Hypertension 66, 00-00.

3. Zhu HD, Pollock NK, Kotak I, et al. (2014) Pediatrics 133. 